\title{
MINIMALLY INVASIVE TREATMENT OF UROGENITAL PROLAPSE USING AMI I-STITCH
}

\author{
Adrian Hasegan, MD, Phd, Nicolae Grigore, MD, Phd, Valentin Pirvut, MD, Ionela Mihai, MD \\ Department of Urology Faculty of Medicine, "Lucian Blaga" University, Sibiu
}

\begin{abstract}
Introduction. Genital prolapse is a pelvic static disorder, which represents the descending of the vaginal walls and the uterus in the vaginal axis with their exteriorization through vaginal introit. It is a public health problem, with a risk of occurrence of $30-40 \%$ in women over 40 years old, of $20-30 \%$ in multiparous, of about $20 \%$ after surgeries, having as the main cause injuries to pelvic supporting and suspension structures. The objective of this paper is to compare the classic transvaginal surgical technique of mounting the prosthesis to the bilateral sacrospinous ligament with the technique of fixing the mesh to the sacrospinous ligament using the I-Stitch device.

Materials and methods. Between 2010 and 2017, in the Sibiu Urology Clinic, there were diagnosed 57 patients $(p)$ with urogenital prolapse who benefited from colpopexy by fixing the polypropylene mesh to the sacrospinous ligament using the I-Stitch device and colpopexy by classically fixing the mesh.

Results. In the group using the I-Stich device, mean operative time was 48.5 minutes (between 35 and 110 minutes), mean blood loss was $140 \mathrm{ml}(90-300 \mathrm{ml})$, postoperative pain present in $5 \mathrm{p}(8.77 \%)$, hospitalization length between 2 and 5 days with an average of 3.2 days.

Conclusion. Reconstructive surgery of pelvic floor is a combination of surgical procedures designed to correct multiple defects, is definitive and curative in the treatment of prolapse.
\end{abstract}

Keywords: genital prolapse, sacrospinous ligament, I-Stitch device, colpopexy

\section{INTRODUCTION}

Sacrospinous colpopexy was introduced by Randall and Nichols in 1971 and has become a favourite method for restoring vaginal support in women with genital prolapse (1).

Genital prolapse is a pelvic static disorder, which represents the descending of the vaginal walls and the uterus in the vaginal axis with their exteriorization through vaginal introit. It is a public health problem, with a risk of occurrence of 30 $40 \%$ in women over 40 years old, of $20-30 \%$ in multiparous, of about $20 \%$ after surgeries, having as the main cause injuries to pelvic supporting and suspension structures. Suspension and supporting structures may be affected by pregnancy, laborious births, pelvic peritoneal muscular-connective defects, estrogenic deficiency, chronic constipation, obesity, perineal surgery $(2,3)$.

Olsen et al. show that $11.1 \%$ of women under the age of 80 may have an intervention for genital prolapse, and reinterventions may reach up to $29 \%$
(4). Fixing the vaginal vault to the sacrospinous ligament is an effective method of restoring vaginal support in women with genital prolapse. Treatment options include: non-surgical methods - pelvic floor rehabilitation, hormonal therapy, Pesar, surgical methods.

The options include transabdominal techniques: classical or laparoscopic sacrofixation, classical or laparoscopic hysterectomy; transvaginal techniques: sacrospinous ligament colpopexy, anterior colporrhaphy, posterior colporrhaphy, vaginal hysterectomy (laparoscopically assisted), treatment of stress urinary incontinence (TOT, TVT), cervical amputation, colpoplasty.

Sacrospinous ligament fixation is the most physiological procedure capable of repositioning the vaginal canal over the levator muscles, mimicking the natural support. During abdominal pressure increase, the vagina is compressed on the levator floor, which prevents it from sliding inside the urogenital hiatus. Although the sacrospinous ligament fixation procedure restores a slightly posteriorly 
oriented vaginal axis, it provides an anatomical better result than sacrocolpopexy to the promontory, which tends to verticalize the vagina. Such verticalization exposes the vagina for secondary hernia in the pelvic floor, such as the enterocele or rectocele (5).

Genital prolapse may be asymptomatic if it is of small size, sometimes patients complain about: urinary stress incontinence, dysuria, polakiuria, urgency-related micturition, incomplete urinary retention, defecation disorders - chronic constipation, seldom faecal incontinence, sexual dysfunction, dyspareunia, incontinence during intercourse, pelvic pressure, pelvic pain, pelvic weight sensation (6).

\section{OBJECTIVES}

The objective of this paper is to compare the classic transvaginal surgical technique of mounting the prosthesis to the bilateral sacrospinous ligament with the technique of fixing the mesh to the sacrospinous ligament using the I-Stich device.

\section{MATERIALS AND METHODS}

Between 2010 and 2017, in the Sibiu Urology Clinic, there were diagnosed 57 patients (p) with urogenital prolapse who benefited from colpopexy by fixing the polypropylene mesh to the sacrospinous ligament using the I-Stitch device and colpopexy by classically fixing the mesh. The classical fixation technique was applied to $22 \mathrm{p}(38.5 \%)$ and the A.M.I. I-Stitch device was used in $35 \mathrm{p}$ $(61.6 \%)$.

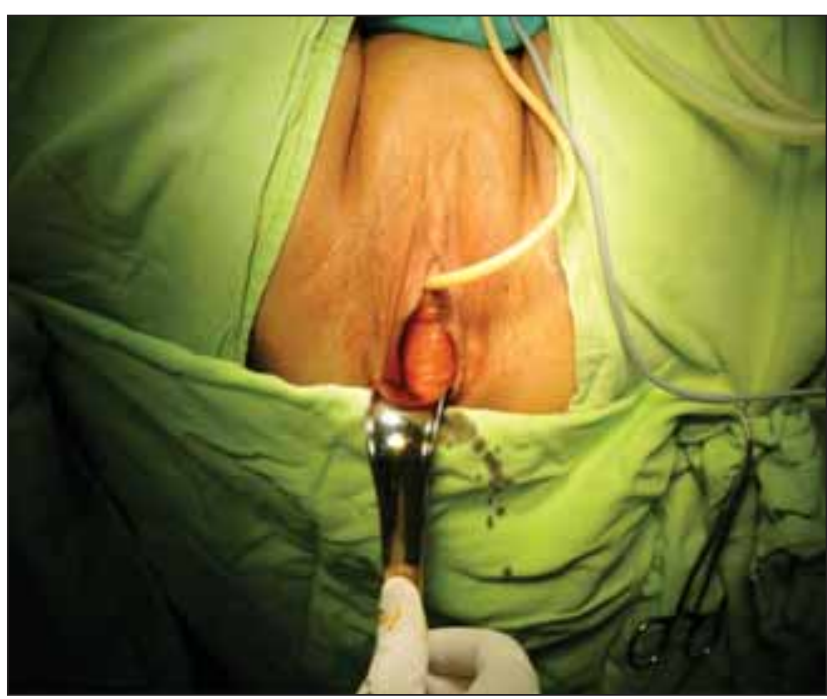

FIGURE 1. Genital prolapse grade 2

The average age of patients was 58.4 years (between 46 and 86 years old). Genital prolapse grade
$18(31.57 \%)$ - (Fig. 1), grade 3-14 p (24.56\%), grade 4-8 p (14.1\%) (Fig. 2). Associated urinary disorders: stress urinary incontinence $-42 \mathrm{p}(73.6 \%)$ of which $14 \mathrm{p}(24.56 \%)$ had occult incontinence, renal failure by chronic retention of urine with bilateral ureterohydronephrosis - $15 \mathrm{p}(26,4 \%)$. Defecation disorders showed $15 \mathrm{p}(26.31 \%)$ and $16 \mathrm{p}$ (28.1\%) showed dyspareunia, concomitant hysterectomy was performed in $22 \mathrm{p}(38.5 \%)$, and $17 \mathrm{p}$ $(29.8 \%)$ had a history of hysterectomy.

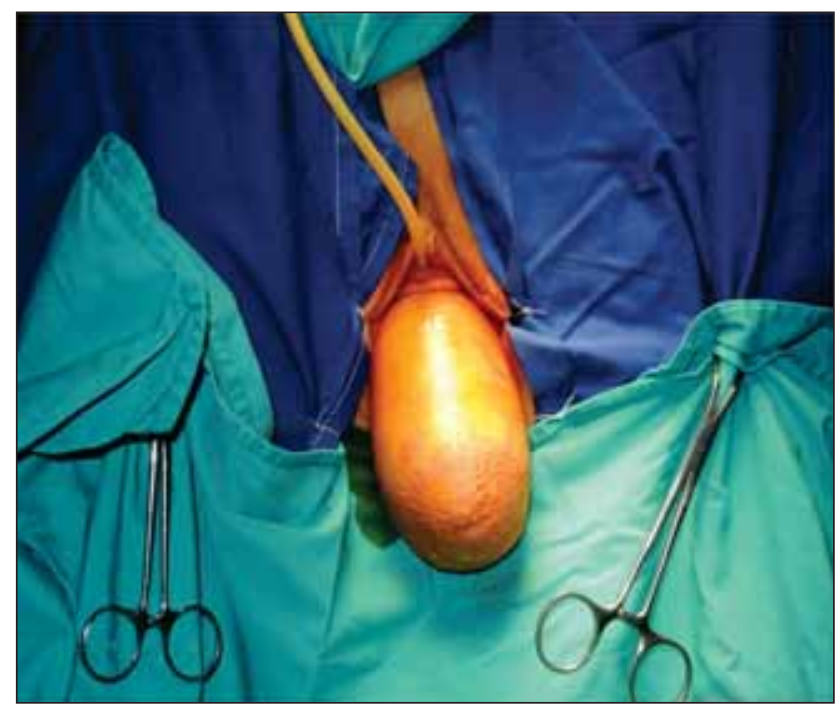

FIGURE 2. Genital prolapse grade 4

\section{RESULTS}

The operations were performed with the patient in the lithotomy position, under spinal anesthesia. In patients with marked genital prolapse, vaginal hysterectomy was the first. Sacrospinous colpospexy actually begins with the hydrodissection of the vaginal floor by injecting saline or xylene with adrenaline $1 / 100,000$. The incision of the posterior vaginal wall from the vault to the vaginal opening is then practiced. The bilateral pararectal space is dissected. The right ischial spine is palpated and a window is created between it and the rectovaginal space using the sharp or blunt dissection. Through the created window, the dissection proceeds to the level of sacrospinous ligament. The suture thread is placed at the sacrospinous ligament one finger medially to the spine to avoid damaging the pudendal plexus. Classically, this technique involves direct viewing of sacrospinous ligament with Breisky valves and fixing the thread with a long porthole (Fig. 3). Using the I-Stich device, pararectal dissection is minimal. The device is guided by using the index to the position of fixing the thread to the sacrospinous ligament (Fig. 4). At this point, the instrument is pressed into the tissue and the tip of the 
loading unit is pushed forward. The loading unit is pushed until a click is heard and the stitch thread is secured in place. With the suture firmly attached to the tissue, the loading unit can be withdrawn with complete removal of the device (Fig. 5). The result is the secure suture fixing in a very difficult position in less than a minute.

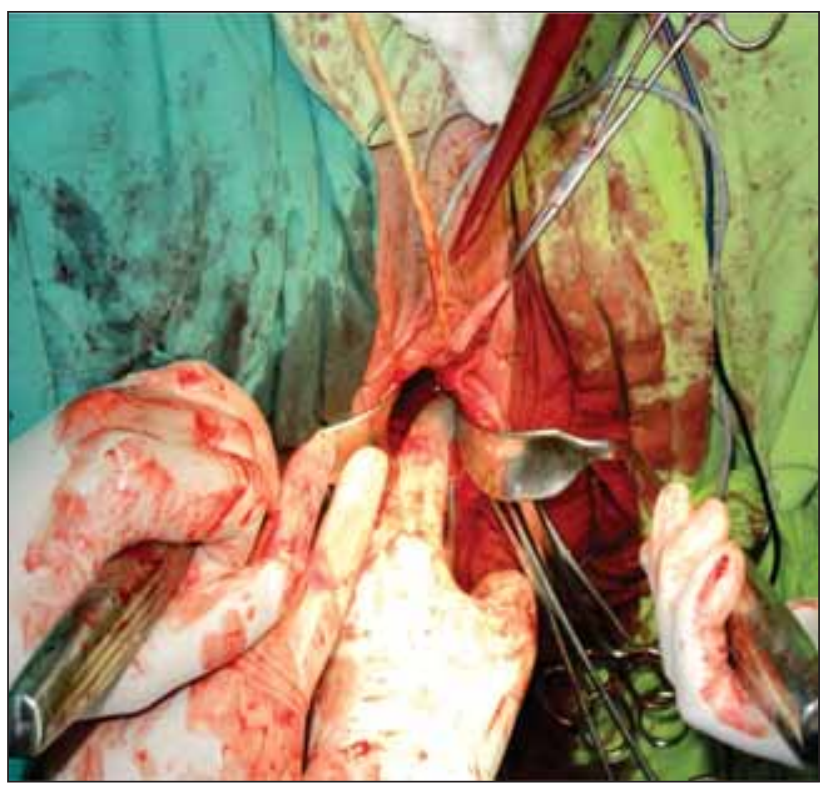

FIGURE 3. Intraoperative image: classicall technique using Breisky valves

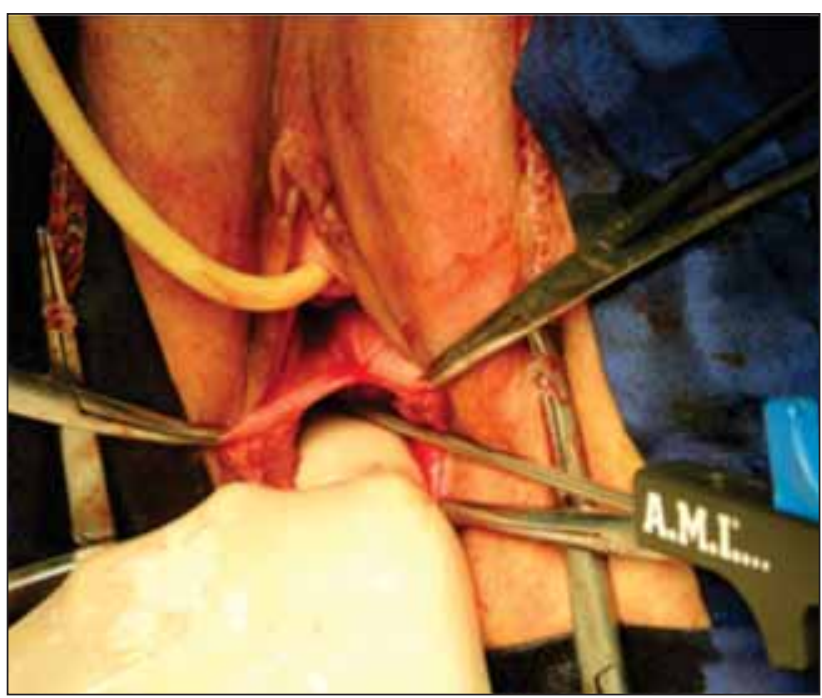

FIGURE 4. Introperative image: I-Stitch device is guided by using the index

After the suture threads are mounted bilaterally, the polypropylene mesh of various shapes is used. The position of the mesh is dependent on the defect to be corrected: anterior, lateral, posterior. The proximal end of the mesh is fixed to the cervix or vaginal vault, restoring the vaginal axis (Fig. 6,7).

The position of the polypropylene mesh is checked. Suture of the vagina's posterior wall with resorbable separate threads is then practiced (Fig. 8).

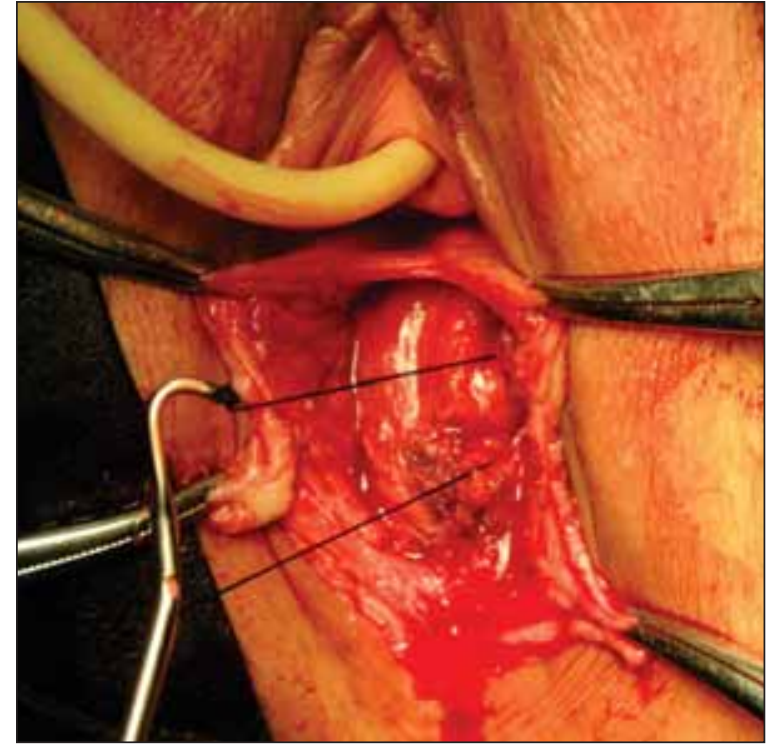

FIGURE 5. Introperative image: the suture attached to the sacrospinous ligament
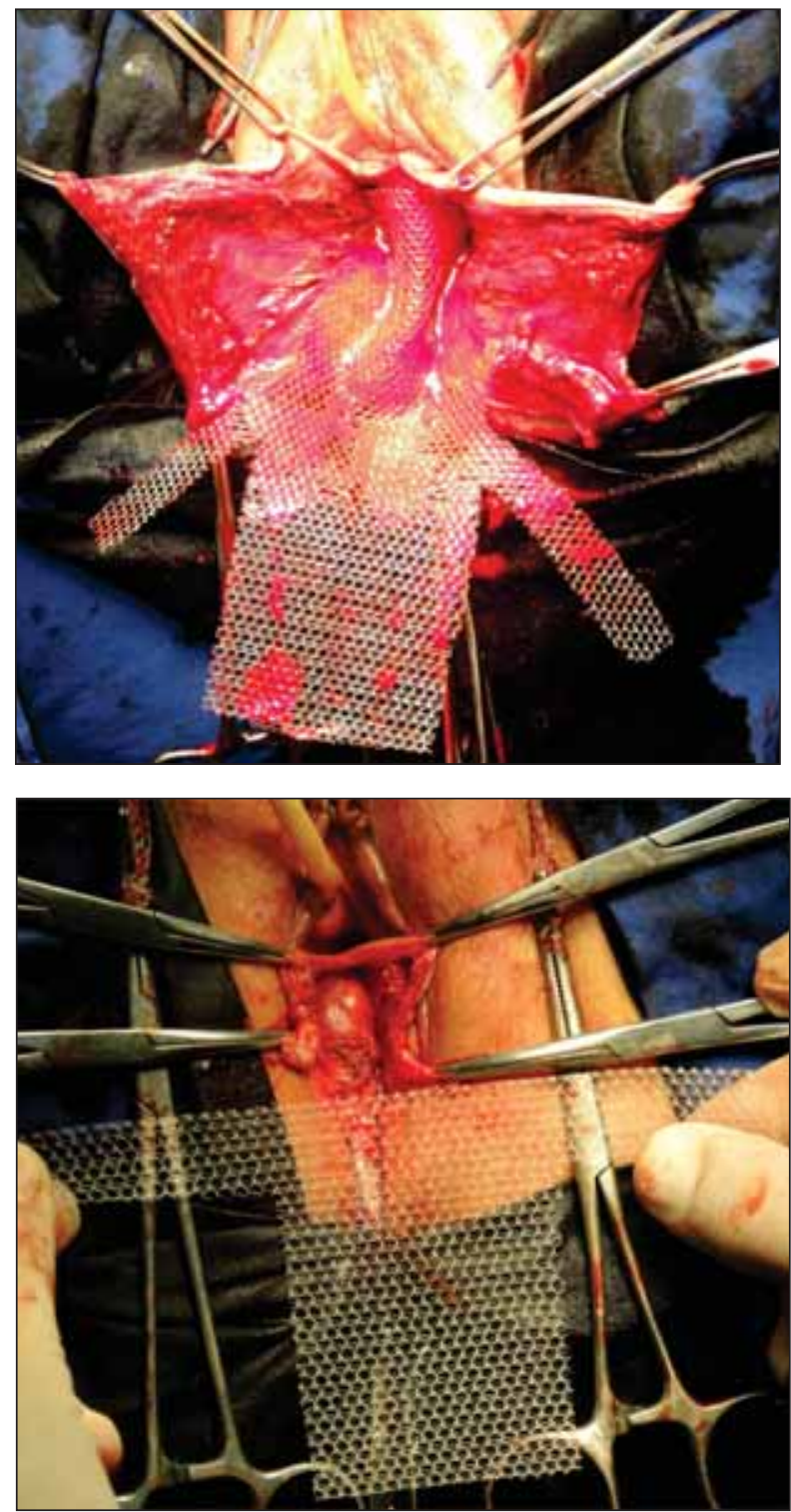

FIGURE 6,7. Introperative image: the position of the mesh 


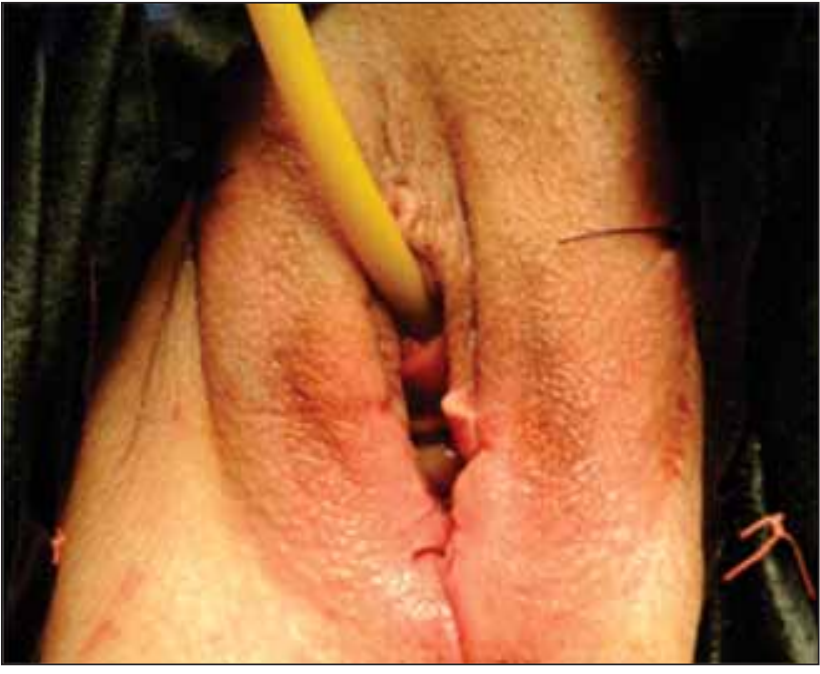

FIGURE 8. Intraoperative image: final aspect

TABLE 1.

\begin{tabular}{|l|c|c|}
\hline $\begin{array}{l}\text { Intra and postoperative } \\
\text { results }\end{array}$ & $\begin{array}{c}\text { Classical } \\
\text { tehnicque }\end{array}$ & AMI I-Stich \\
\hline Mean operative time $(\mathrm{min})$ & $\begin{array}{c}70.5 \\
(60-140 \mathrm{~min})\end{array}$ & $\begin{array}{c}48.5 \\
(35-110 \mathrm{~min})\end{array}$ \\
\hline Mean blood loss (ml) & $\begin{array}{c}270 \\
(180-530 \mathrm{ml})\end{array}$ & $\begin{array}{c}140 \\
(90-300 \mathrm{ml})\end{array}$ \\
\hline Postoperative pain $(\mathrm{p})$ & $15 \mathrm{p} \mathrm{(26.31 \% )}$ & $5 \mathrm{p} \mathrm{(8.77 \% )}$ \\
\hline Hospitalization stay (days) & $4.1(4-6$ days) & $3.2(2-5$ days) \\
\hline $\begin{array}{l}\text { Postoperative } \\
\text { complications }\end{array}$ & $3 \mathrm{p} \mathrm{(5.2 \% )}$ & $2 \mathrm{p} \mathrm{(3.5 \% )}$ \\
\hline Urinary tract infection & $1 \mathrm{p} \mathrm{(1.75 \% )}$ & - \\
\hline $\begin{array}{l}\text { Persistent stress urinary } \\
\text { incontinence }\end{array}$ & - & $1(1.75 \%)$ \\
\hline $\begin{array}{l}\text { Incomplete urinary } \\
\text { retention }\end{array}$ & & \\
\hline
\end{tabular}

In the group where the classical technique was practiced, mean operative time was 70.5 minutes (between 60 minutes and 140 minutes), mean blood loss was $270 \mathrm{ml}(180-530 \mathrm{ml})$, postoperative pain was present in $15 \mathrm{p}(26,31 \%)$, the length of hospitalization was between 4 and 6 days with an average of 4.1 days.

In the group using the I-Stich device, mean operative time was 48.5 minutes (between 35 and 110 minutes), mean blood loss was $140 \mathrm{ml}$ (90-300 ml), postoperative pain present in $5 \mathrm{p}(8.77 \%)$, hospitalization length between 2 and 5 days with an average of 3.2 days.

Treatment of stress urinary incontinence (SUI) with subcutaneous TOT fixation was performed during the same intervention in all patients with SUI present (42 p, 73.6\%).

Ablation of the urethrovesicle catether was performed second days postoperatively.

Intra and post-operative complications cited in the literature include: bladder, rectal injury, pudendal vascular network injury or sacral vein injury leading to haemorrhage with subsequent pelvic hematoma, nerve injury involving buttock pain, dyspareunia, urinary tract infection, urinary retention, stress urinary incontinence, wound infection, ischiorectal fossa abscess, erosion of the vaginal wall. Extremely rare complications: myositis in the buttock region, evisceration (when hysterectomy is also associated) (7).

Postoperative complications were recorded in $7 \mathrm{p}$ $(12.28 \%)$, of which $5 \mathrm{p}(8.7 \%)$ had urinary tract infection, treated with antibiotics, $1 \mathrm{p}(1.75 \%)$ had persistent stress urinary incontinence, incomplete urinary retention in 1 patient $(1.75 \%)$ resolved by elongation of the tape during the first 10 days postoperatively.

Postoperative follow-up was 18 months (between 12-40 months) on average.

\section{DISCUSSIONS}

Sacrospinous colpopexy should be performed after careful clinical examination, completion of investigations and treatment of associated conditions (acute renal failure, chronic incomplete urinary retention, coagulopathy, sepsis).

The modern technique of fixing the polypropylene mesh to the bilateral sacrospinous ligament using the I-Stich device has reduced operative time by approximately 40 minutes, compared to the classical method that uses the valves (48.5 minutes/ 70.5 minutes).

A lower intraoperative bleeding average was found for the method using the I-Stich device (140 $\mathrm{ml})$ versus the classical technique $(270 \mathrm{ml})$.

Administration of anti-inflammatory drugs was made for a shorter period of time in the patients who were operated by the modern technique ( $5 \mathrm{p}-$ $8.77 \%$ ), compared to those who were operated by the classical technique $(15 \mathrm{p}-26.31 \%)$.

A shorter period of hospitalization was recorded in the group using the I-Stich, compared to the group where the valves (3.2/4.1) were used.

A review of five observational studies reported that combined sacral colpopexy and paravaginal repair successfully treated anterior prolapse in 76 to 97 percent of women (8).

Repair of anterior or posterior prolapse alone appears to have a higher failure rate than when these procedures are combined with apical prolapse repair. This was illustrated in United States national study of 2,756 women and found the following 10year reoperation rates: anterior repair versus combined anterior and apical repair (20.2 versus 11.6 percent); anterior and posterior repair versus com- 
bined anterior, posterior, and apical repair (14.7 versus 10.2 percent); and posterior repair versus combined posterior and apical repair (14.6 versus 12.9 percent) (9).

Advantages of uterine sparing techniques are: a shorter operative duration and less blood loss; however, their efficacy is controversial (10-14). Two randomized trials in women with stage II or higher POP that compared transvaginal sacrospinous hysteropexy with vaginal hysterectomy (with uterosacral or sacrospinous ligament suspension of the vaginal vault) bring consistent results: the rate of prolapse recurrence after 9 to 12 months was higher in women who underwent hysteropexy in both trials, but reached statistical significance in one trial (27 versus 3 percent) (13) and not the other (25 versus 13 percent) (14). Operative duration (59 versus 120 minutes in one trial) and blood loss (20 versus $120 \mathrm{ml}$ in one trial) were decreased for sacrospinous hysteropexy compared with vaginal hysterectomy; complication rates were similar for the two groups (14).

Benson et al. conducted a prospective randomized study of vaginal versus abdominal reconstructive surgery for the treatment of pelvic support defects, and found that the abdominal approach was more effective for correction of significant pelvic support defects. By contrast with Hardiman and Drutz, who found that sacrospinous fixation and abdominal colposacropexy have equal efficiency.

\section{REFERENCES}

1. Randall C.L., Nichols D.H. Surgical treatment of vaginal inversion. Obstet Gynecol. 1971; 38:327-32. PubMed PMID: 5094313.

2. Olsen A.L., Smith V.J., Bergstrom J.O., et al. Epidemiology of surgically managed pelvic organ prolapse and urinary incontinence. Obstet. Gynecol 1997, 89-501

3. Asante A., Whiteman M.K., Kulkami A., et al. Elective oophorectomy in the United States: trens and in-hospital complications, 1998-2006. Obstet Gynecol 2010, 116:1088.

4. Olsen A.L., Smith V.J., Bergstrom J.O., Colling J.C., Clark A.L. Epidemiology of surgically managed pelvic organ prolapse and urinary incontinence. Obstet Gynecol. 1997 Apr; 89(4):501-6).

5. Klutke C.G., Siegel C.L. Functional female pelvic anatomy. Urol Clin North Am 1995; 22:487-498.

6. DeLancey J.O.L. Relationship of prolapse syndromes to symptoms, Kursh E, McGuireE, Female Urology, J.B Lippincott, Philadelphia, 1994:223-229.

7. Petri E., Ashok K. Sacrospinous vaginal Fixation - current status. Acta Obstet Gynecol Scand. 2011; 90:429-436.

8. Maher C., Baessler K. Surgical management of anterior vaginal wall prolapse: an evidence based literature review. Int Urogynecol J Pelvic Floor Dysfunct 2006; 17:195.
The I-Stich device avoids the abdominal time of the promontofixation surgery (classically or laparoscopically), avoids reintervention in the abdomen, allows for the simultaneous resolution of the entire pelvic floor pathology (uterine prolapse, stress urinary incontinence, cystocele, rectocele, elytrocele), reduced postoperative pain (lower antialgic medication), early mobilization, early resumption of digestive transit, short-term hospitalization, rapid socio-professional reintegration.

\section{CONCLUSIONS}

Reconstructive surgery of pelvic floor is a combination of surgical procedures designed to correct multiple defects, is definitive and curative in the treatment of prolapse.

The technique of fixing the polypropylene mesh to the bilateral sacrospinous ligament using the I-Stich device is an efficient and rapid treatment, with a low risk of recurrence and complications, allowing the cure of associated urinary tract disorders during the same intervention.

Acknowledgements: The authors declare no conflict of interests and no sponsorship was provided by the manufacturer of the catheters involved in this study. All authors have read and approved this publication and had equal scientific contribution in publishing this material.
9. Eilber K.S., Alperin M., Khan A., et al. Outcomes of vaginal prolapse surgery among female Medicare beneficiaries: the role of apical support. Obstet Gynecol 2013; 122:981.

10. Hefni M., El-Toukhy T., Bhaumik J., Katsimanis E. Sacrospinous cervicocolpopexy with uterine conservation for uterovaginal prolapse in elderly women: an evolving concept. Am J Obstet Gynecol 2003; 188:645.

11. Maher C.F., Cary M.P., Slack M.C., et al. Uterine preservation or hysterectomy at sacrospinous colpopexy for uterovaginal prolapse? Int Urogynecol J Pelvic Floor Dysfunct 2001; 12:381.

12. Diwan A., Rardin C.R., Kohli N. Uterine preservation during surgery for uterovaginal prolapse: a review. Int Urogynecol J Pelvic Floor Dysfunct 2004; 15:286.

13. Carramão S., Auge A.P., Pacetta A.M., et al. [A randomized comparison of two vaginal procedures for the treatment of uterine prolapse using polypropylene mesh: hysteropexy versus hysterectomy]. Rev Col Bras Cir 2009; 36:65.

14. Dietz V., van der Vaart C.H., van der Graaf Y., et al. One-year follow-up after sacrospinous hysteropexy and vaginal hysterectomy for uterine descent: a randomized study. Int Urogynecol J 2010; 21:209. 